\title{
Classification of Bipartite and Tripartite Qutrit Entanglement under SLOCC
}

\author{
Xin-Gang Yang ${ }^{1}$, Zhi-Xi Wang ${ }^{1}$, Xiao-Hong Wang $^{1}$ and Shao-Ming Fei ${ }^{1,2}$ \\ ${ }^{1}$ College of Mathematical Scicences, Capital Normal University, Beijing 100037, \\ China \\ ${ }^{2}$ Max-Planck-Institute for Mathematics in the Sciences, 04103 Leipzig, Germany
}

\begin{abstract}
We classify biqutrit and triqutrit pure states under stochastic local operations and classical communication. By investigating the right singular vector spaces of the coefficient matrices of the states, we obtain explicitly two equivalent classes of biqutrit states and twelve equivalent classes of triqutrit states respectively.
\end{abstract}

Keywords: biqutrit, triqutrit, SLOCC

PACS: 03.67.Mn, 03.65.Ud, 02.10.Yn

\section{Introduction}

It is well known that quantum entanglement plays very important roles in quantum information theory. One of the main tasks in quantum information theory is to find out how many different ways multipartite pure states can be entangled. It has been shown that entangled qudits are less affected by noise than entangled qubits [1, 2]. In quantum cryptography it is more secure against evesdropping attacks using entangled qutrits or qudits than using qubits [3, 4, 5, 6]. These facts motivate our interest in multi-dimensional entangled states.

As the concept of entanglement is related to the nonlocal properties of a state, local quantum operations can not affect the intrinsic nature of entanglement[7]. It is natural and meaningful to classify pure states in terms of stochastic local operations and classical communication (SLOCC). In [8] it was shown that SLOCC equivalent pure states can carry out the same quantum-informational tasks with non-null possibly different possibilities, and two $N$-partite states $\Psi$ and $\Phi$ are equivalent under SLOCC if and only if there exist invertible local operations (ILOs) $F^{[1]}, \cdots, F^{[N]}$ such that $\Psi=F^{[1]} \otimes F^{[2]} \otimes \cdots \otimes F^{[N]} \Phi$.

In recent years, a lot of efforts have been made on classification of multipartite entanglement under SLOCC [3, 9-18]. In [15] an inductive method of classifying $n$-qubit entanglement under SLOCC has been presented, from which the entanglement classifica- 
tion of three and four qubits have been obtained. In the classification of biqutrit pure states some entanglement measures have been also used in [3]. In [9] a range criterion has been used to judge the equivalence of two states under SLOCC. The classification of entanglement in $2 \times m \times n$ systems is investigated. In [10] the complete SLOCC classification of multipartite entanglement in $2 \times 2 \times n$ cases has been studied in two different ways. It has been proved that a pure state of four qubits can be transformed into nine families by SLOCC operations with determinant one [13]. In [14] three-qubit states under SLOCC on the basis of the canonical forms and on the local unitary operator polynomial invariants have been classified.

In this paper we study the classification of biqutrit and triqutrit pure states under SLOCC by using the method introduced in [15]. According to the dimensions of the right singular vector spaces of the coefficient matrices of the states, we obtain explicitly equivalent classifications of biqutrit states and triqutrit states under SLOCC.

\section{Classification of biqutrit entanglement}

Let $\left\{e_{i}\right\}_{i=1, \ldots, m}$ and $\left\{f_{j}\right\}_{j=1, \ldots, n}$ denote bases in $\mathbb{C}^{m}$ and $\mathbb{C}^{n}$, respectively. Any bipartite state $\Psi \in C^{m} \otimes C^{n}$ can be written as

$$
\Psi=\sum_{i=1}^{m} \sum_{j=1}^{n} c_{i j} e_{i} \otimes f_{j},
$$

where $c_{i j} \in \mathbb{C}$. We denote $C=\left(c_{i j}\right)_{m, n}$ the coefficient matrix of the state $\Psi$.

According to the singular value decomposition, an $m \times n$ matrix $C$ can always be decomposed as $C=V \Sigma W^{\dagger}$, where $V$ and $W$ are unitary matrices and $\Sigma$ is a diagonal matrix with non-negative entries (singular values), $\Sigma_{i j}=\sigma_{i} \delta_{i j}, i=1, \ldots, m, j=1, \ldots, n$ and $\sigma_{k} \geq 0$ for all $k$. The columns $v_{i}$ of $V=\left[\begin{array}{llll}v_{1} & v_{2} & \ldots & v_{m}\end{array}\right]$ (resp. $w_{i}$ of $W=$ $\left.\left[\begin{array}{llll}w_{1} & w_{2} & \ldots & w_{n}\end{array}\right]\right)$ are called left (resp. right) singular vectors of $C$.

Since the relevant singular vectors will be those associated to non-null singular values, in the following, we agree on referring as singular vectors only to those $v_{k}$ and $w_{k}$ for which $\sigma_{k}>0$. We denote by $\Gamma$ (resp. $\Pi$ ) the subspace generated by the left (resp. right) singular vectors, i.e. $\Gamma=\operatorname{span}\left\{v_{1}, \ldots, v_{k}\right\}$ (resp. $\left.\Pi=\operatorname{span}\left\{w_{1}, \ldots, w_{k}\right\}\right)$. From the Schmidt decomposition of bipartite pure states, the state (11) is separable if and only if $\operatorname{dim} W=1$ (or $\operatorname{dim} V=1)$ [15].

Let $\Psi, \bar{\Psi} \in \mathbb{C}^{m} \otimes \mathbb{C}^{n}$ denote two bipartite states related by SLOCC, i.e.

$$
\bar{\Psi}=F^{[1]} \otimes F^{[2]}(\Psi)
$$


where $F^{[1]}$ and $F^{[2]}$ are non-singular operators upon $\mathbb{C}^{m}$ and $\mathbb{C}^{n}$, respectively. Similar to the two-qubit case [15], in terms of singular value decomposition one can prove that the corresponding coefficient matrices $C$ of $\Psi, \bar{C}$ of $\bar{\Psi}$ in an arbitrary product basis are related through

$$
\bar{C}=\left(F^{[1]^{T}} V\right) \Sigma\left(F^{[2] \dagger} W\right)^{\dagger},
$$

i.e. if $v_{j}$ and $w_{j}$ are the left and right singular vectors of the coefficient matrix $C$ respectively, then the new left and right singular vectors with respect to $\bar{C}$ will be $F^{[1]^{T}}\left(v_{j}\right)$ and $F^{[2] \dagger}\left(w_{j}\right)$ respectively.

For simplicity, we will write it as $\bar{\Psi}=F^{[1]^{T}} \otimes F^{[2]^{\dagger}}(\Psi)$ in stead of (2). We first consider the biqutrit $(n=m=3)$ case. In this case, the coefficient matrix of an arbitrary pure state in $\mathbb{C}^{3} \otimes \mathbb{C}^{3}$ can be expressed as

$$
C=\left(\begin{array}{lll}
C_{11} & C_{12} & C_{13} \\
C_{21} & C_{22} & C_{23} \\
C_{31} & C_{32} & C_{33}
\end{array}\right)
$$

The dimensions of the right singular subspaces $\Pi$ could be 1,2 or 3 .

If $\operatorname{dim} \Pi=1$, we can choose ILOs $F^{[1]}$ and $F^{[2]}$ such that

$$
F^{[1]}\left(v_{1}\right)=\frac{1}{\sigma_{1}} e_{1}, \quad F^{[2]}\left(w_{1}\right)=e_{1},
$$

where $\left\{e_{1}, e_{2}, e_{3}\right\}$ denote bases of $\mathbb{C}^{3}$. The new coefficient matrix $\bar{C}$ is then of the form

$$
\bar{C}=\left(\begin{array}{lll}
1 & 0 & 0 \\
0 & 0 & 0 \\
0 & 0 & 0
\end{array}\right),
$$

which correspond to the product state $\Psi_{0}=|00\rangle$ (we denote $e_{1}=|0\rangle, e_{2}=|1\rangle$ and $e_{3}=|2\rangle$ as usual in the following).

In the case $\operatorname{dim} \Pi=2$, we choose ILOs $F^{[1]}$ and $F^{[2]}$ such that

$$
F^{[1]}\left(v_{1}\right)=\frac{1}{\sigma_{1}} e_{1}, \quad F^{[1]}\left(v_{2}\right)=\frac{1}{\sigma_{2}} e_{2}, \quad F^{[2]}\left(w_{1}\right)=e_{1}, \quad F^{[2]}\left(w_{2}\right)=e_{2} .
$$

The new coefficient matrix will turn to be

$$
\bar{C}=\left(\begin{array}{lll}
1 & 0 & 0 \\
0 & 1 & 0 \\
0 & 0 & 0
\end{array}\right),
$$

which correspond to the state $\Psi_{1}=|00\rangle+|11\rangle$. 
For the case $\operatorname{dim} \Pi=3$, we choose $\operatorname{ILOs} F^{[1]}$ and $F^{[2]}$ such that

$$
\begin{array}{r}
F^{[1]}\left(v_{1}\right)=\frac{1}{\sigma_{1}} e_{1}, \quad F^{[1]}\left(v_{2}\right)=\frac{1}{\sigma_{2}} e_{2}, \quad F^{[1]}\left(v_{3}\right)=\frac{1}{\sigma_{3}} e_{3}, \\
F^{[2]}\left(w_{1}\right)=e_{1}, \quad F^{[2]}\left(w_{2}\right)=e_{2}, \quad F^{[2]}\left(w_{3}\right)=e_{3} .
\end{array}
$$

Then $\bar{C}$ turns out to be a $3 \times 3$ identity matrix, and the corresponding state is $\Psi_{2}=$ $|00\rangle+|11\rangle+|22\rangle$.

Therefore, biqutrit states can be entangled in two inequivalent ways ( $\Psi_{1}$ and $\left.\Psi_{2}\right)$ under SLOCC. While in [3] biqutrit entangled states are classified into three types:

$$
\begin{aligned}
|I\rangle & =\frac{1}{\sqrt{2}}(|11\rangle+|00\rangle), \quad|I I\rangle=\frac{1}{\sqrt{3}}(|11\rangle+|00\rangle+|-1-1\rangle), \\
|I I I\rangle & =\frac{1}{\sqrt{6}}(|11\rangle+|-1-1\rangle+|10\rangle+|01\rangle+|0-1\rangle+|-10\rangle) .
\end{aligned}
$$

We find that the type $|I I\rangle$ is in fact equivalent to the type $|I I I\rangle$ under SLOCC: $|I I I\rangle$ can written as

$|I I I\rangle=\frac{1}{\sqrt{3}}\left[|1\rangle \otimes \frac{1}{\sqrt{2}}(|1\rangle+|0\rangle)+|0\rangle \otimes \frac{1}{\sqrt{2}}(|1\rangle+|-1\rangle)+|-1\rangle \otimes \frac{1}{\sqrt{2}}(|-1\rangle+|0\rangle)\right]$,

by choosing the ILOs

$$
F^{[1]}=\left(\begin{array}{ccc}
1 & 0 & 0 \\
0 & 1 & 0 \\
0 & 0 & 1
\end{array}\right), \quad F^{[2]}=\frac{1}{\sqrt{2}}\left(\begin{array}{ccc}
1 & 1 & -1 \\
1 & -1 & 1 \\
-1 & 1 & 1
\end{array}\right)
$$

we get $F^{[1]} \otimes F^{[2]}|I I I\rangle=|I I\rangle$. This result can be also obtained by using the method of Schmidt decomposition provided in [8].

\section{Classification of triqutrit entanglement}

An arbitrary triqutrit pure state $\Psi \in \mathbb{C}^{3} \otimes \mathbb{C}^{3} \otimes \mathbb{C}^{3}$ has the form

$$
\Psi=\sum_{i, j, k=1}^{3} c_{i j k} e_{i} \otimes e_{j} \otimes e_{k} .
$$

We can write the coefficient matrix of $\Psi$ in the form

$$
C=C_{1 \mid 23}=\left(\begin{array}{lllllllll}
C_{111} & C_{112} & C_{113} & C_{121} & C_{122} & C_{123} & C_{131} & C_{132} & C_{133} \\
C_{211} & C_{212} & C_{213} & C_{221} & C_{222} & C_{223} & C_{231} & C_{232} & C_{233} \\
C_{311} & C_{312} & C_{313} & C_{321} & C_{322} & C_{323} & C_{331} & C_{332} & C_{333}
\end{array}\right) .
$$


There are also two other ways to write the coefficient matrix of $\Psi: C_{2 \mid 13}$ and $C_{3 \mid 12}$. Without loss of generality, we will use $C_{1 \mid 23}$ in below considerations.

The classification of triqutrit pure states is to choose the ILOs $F^{[1]}, F^{[2]}$ and $F^{[3]}$ such that the final coefficient matrix reduces to a canonical one. In order to do so, we have to find all possible structures of the space $\Pi$.

Let $\Psi, \bar{\Psi} \in \mathbb{C}^{m} \otimes \mathbb{C}^{n} \otimes \mathbb{C}^{l}$ denote two tripartite states that are equivalent under SLOCC, i.e.

$$
\bar{\Psi}=F^{[1]^{T}} \otimes\left(F^{[2]} \otimes F^{[3]}\right)^{\dagger}(\Psi)
$$

where $F^{[1]}, F^{[2]}$ and $F^{[3]}$ are non-singular operators upon $\mathbb{C}^{m}, \mathbb{C}^{n}$ and $\mathbb{C}^{l}$, respectively. Similarly, we can prove that the coefficient matrices $C$ and $\bar{C}$ in an arbitrary product basis are related through

$$
\bar{C}=\left(F^{[1]} V\right) \Sigma\left(F^{[2]} \otimes F^{[3]} W\right)^{\dagger}
$$

Concerning the dimension of the right singular subspace $\Pi$, there are again three possibilities $(\operatorname{dim} \Pi=1, \operatorname{dim} \Pi=2$ and $\operatorname{dim} \Pi=3)$ for triqutrit case.

\subsection{The case of $\operatorname{dim} \Pi=1$}

1. Case $\Pi=\operatorname{span}\left\{\Psi_{0}\right\}$, where $\Psi_{0}$ is the product state defined in the last section. In this case, $w_{1}$ is of the form $w_{1}=\phi \otimes \psi$. We can choose ILOs $F^{[1]}, F^{[2]}$ and $F^{[3]}$ such that

$$
F^{[1]}\left(v_{1}\right)=\frac{1}{\sigma_{1}} e_{1}, \quad F^{[2]}(\phi)=e_{1}, \quad F^{[3]}(\psi)=e_{1} .
$$

Then the new coefficient matrix is of the form

$$
\begin{aligned}
\bar{C} & =\left(\begin{array}{ccc}
\frac{1}{\sigma_{1}} & \cdot & \cdot \\
0 & \cdot & \cdot \\
0 & \cdot & \cdot
\end{array}\right)\left(\begin{array}{cccc}
\sigma_{1} & 0 & 0 & \\
0 & 0 & 0 & \\
0 & 0 & 0
\end{array}\right)\left(\begin{array}{ccccccccc}
1 & 0 & 0 & 0 & 0 & 0 & 0 & 0 & 0 \\
\cdot & \cdot & \cdot & \cdot & \cdot & \cdot & \cdot & \cdot & \cdot \\
\cdot & \cdot & \cdot & \cdot & \cdot & \cdot & . & \cdot & \cdot
\end{array}\right) \\
& =\left(\begin{array}{ccccccccc}
1 & 0 & 0 & 0 & 0 & 0 & 0 & 0 & 0 \\
0 & 0 & 0 & 0 & 0 & 0 & 0 & 0 & 0 \\
0 & 0 & 0 & 0 & 0 & 0 & 0 & 0 & 0
\end{array}\right),
\end{aligned}
$$

which corresponds to the state $e_{1} \otimes e_{1} \otimes e_{1} \equiv|000\rangle$, and where the dots $\cdot$ indicates the irrelevant character of that entry.

2. Case $\Pi=\operatorname{span}\left\{\Psi_{1}\right\}$. In this case, the vector $w_{1}=\phi_{1} \otimes \psi_{1}+\phi_{2} \otimes \psi_{2}$. Using the same strategy as above, we obtain the state $e_{1} \otimes e_{1} \otimes e_{1}+e_{1} \otimes e_{2} \otimes e_{2}$, which corresponds to the canonical vector: $|000\rangle+|011\rangle$. 
3. Case $\Pi=\operatorname{span}\left\{\Psi_{2}\right\}=\operatorname{span}\left\{\phi_{1} \otimes \psi_{1}+\phi_{2} \otimes \psi_{2}+\phi_{3} \otimes \psi_{3}\right\}$. We obtain the state $e_{1} \otimes e_{1} \otimes e_{1}+e_{1} \otimes e_{2} \otimes e_{2}+e_{1} \otimes e_{3} \otimes e_{3}$, which corresponds to the canonical vector: $|000\rangle+|011\rangle+|022\rangle$.

\subsection{The case of $\operatorname{dim} \Pi=2$}

We first consider $\Pi=\operatorname{span}\left\{\Psi_{0}, \Psi_{0}\right\}$. One of the three possible cases in this class is $\Pi=$ $\operatorname{span}\left\{\phi \otimes \psi_{1}, \phi \otimes \psi_{2}\right\}$. In this case, $w_{1}=u_{11} \phi \otimes \psi_{1}+u_{12} \phi \otimes \psi_{2}$ and $w_{2}=u_{21} \phi \otimes \psi_{1}+u_{22} \phi \otimes \psi_{2}$, where the matrix $\left[u_{i j}\right]$ has rank two, since $w_{1}$ and $w_{2}$ are linearly independent. We choose the ILOs $F^{[1]}, F^{[2]}$ and $F^{[3]}$ such that

$$
\begin{array}{r}
\left.F_{1}^{[1]}\left(v_{1}\right)=\frac{1}{\sigma_{1}} e_{1}, \quad F_{1}^{[1]}\left(v_{2}\right)=\frac{1}{\sigma_{2}} e_{2}, \quad F_{2}^{[1]}=\left(\begin{array}{cc}
u_{11}^{*} & u_{12}^{*} \\
u_{21}^{*} & u_{22}^{*} \\
0
\end{array}\right)^{-1} \begin{array}{c}
0 \\
0
\end{array}\right), \\
F^{[1]}=F_{2}^{[1]} F_{1}^{[1]}, \quad F^{[2]}(\phi)=e_{1}, \quad F^{[3]}\left(\psi_{1}\right)=e_{1}, \quad F^{[3]}\left(\psi_{2}\right)=e_{2} .
\end{array}
$$

The new coefficient matrix will be

$$
\begin{aligned}
\bar{C} & =F_{2}^{[1]}\left(\begin{array}{ccc}
\frac{1}{\sigma_{1}} & 0 & . \\
0 & \frac{1}{\sigma_{2}} & \cdot \\
0 & 0 & .
\end{array}\right)\left(\begin{array}{cccc}
\sigma_{1} & 0 & 0 \\
0 & \sigma_{2} & 0 \\
0 & 0 & 0
\end{array}\right)\left(\begin{array}{ccccccccc}
u_{11}^{*} & u_{12}^{*} & 0 & 0 & 0 & 0 & 0 & 0 & 0 \\
u_{21}^{*} & u_{22}^{*} & 0 & 0 & 0 & 0 & 0 & 0 & 0 \\
. & . & . & . & . & . & . & . & .
\end{array}\right) \\
& =\left(\begin{array}{ccccccccc}
1 & 0 & 0 & 0 & 0 & 0 & 0 & 0 & 0 \\
0 & 1 & 0 & 0 & 0 & 0 & 0 & 0 & 0 \\
0 & 0 & 0 & 0 & 0 & 0 & 0 & 0 & 0
\end{array}\right),
\end{aligned}
$$

which corresponds to the state $|000\rangle+|101\rangle$.

Dealting with similarly, we can get two states $|000\rangle+|110\rangle$ and $|000\rangle+|111\rangle$ from the other $\operatorname{cases}\left(\Pi=\operatorname{span}\left\{\phi_{1} \otimes \psi, \phi_{2} \otimes \psi\right\}\right.$ and $\left.\Pi=\operatorname{span}\left\{\phi_{1} \otimes \psi_{1}, \phi_{2} \otimes \psi_{2}\right\}\right)$.

Using the same strategy, all together we obtain the following classifications: 


\begin{tabular}{|c|c|}
\hline Class & Canonical Vector \\
\hline $\operatorname{span}\left\{\Psi_{0}, \Psi_{0}\right\}$ & $|000\rangle+|101\rangle, \quad|000\rangle+|110\rangle$ \\
\hline $\operatorname{span}\left\{\Psi_{1}, \Psi_{1}\right\}$ & $\begin{array}{ll}|000\rangle+|011\rangle+|101\rangle+|112\rangle, & |000\rangle+|011\rangle+|112\rangle+|120\rangle \\
|000\rangle+|011\rangle+|120\rangle+|101\rangle, & |000\rangle+|011\rangle+|120\rangle+|102\rangle\end{array}$ \\
\hline $\operatorname{span}\left\{\Psi_{0}, \Psi_{1}\right\}$ & $\begin{array}{ll}|000\rangle+|011\rangle+|101\rangle, & |000\rangle+|011\rangle+|112\rangle \\
|000\rangle+|011\rangle+|120\rangle, & |000\rangle+|011\rangle+|122\rangle\end{array}$ \\
\hline $\operatorname{span}\left\{\Psi_{0}, \Psi_{2}\right\}$ & $|000\rangle+|011\rangle+|022\rangle+|101\rangle$ \\
\hline $\operatorname{span}\left\{\Psi_{1}, \Psi_{2}\right\}$ & $\begin{array}{l}|000\rangle+|011\rangle+|022\rangle+|101\rangle+|112\rangle \\
|000\rangle+|011\rangle+|022\rangle+|112\rangle+|120\rangle \\
|000\rangle+|011\rangle+|022\rangle+|120\rangle+|101\rangle\end{array}$ \\
\hline
\end{tabular}

We did not consider the case $\Pi=\operatorname{span}\left\{\Psi_{2}, \Psi_{2}\right\}$. This is due to that any twodimensional subspace in $\mathbb{C}^{3} \otimes \mathbb{C}^{3}$ contains at least one product vector $\Psi_{0}$ or one entangled vector $\Psi_{1}$ with coefficient matrix rank two. This can be got by the following way: Let $V$ be a two-dimensional subspace of $\mathbb{C}^{3} \otimes \mathbb{C}^{3}$. Without loss of generality, two entangled vectors of rank 3 can be chosen as generators of $V$ with coefficient matrices given by $C_{1}=I$ and $C_{2}$ being an arbitrary matrix of rank 3 in the product canonical basis. Then it is always possible to find non-null complex numbers $\alpha$ and $\beta$ such that $\alpha I+\beta C_{2}$ has rank one or two: because $-\beta / \alpha$ must be chosen to be an eigenvalues of $C_{2}$, and if two eigenvalue of $C_{2}$ is the same, $\alpha I+\beta C_{2}$ will have rank one.

\subsection{The case of $\operatorname{dim} \Pi=3$}

We first consider $\Pi=\operatorname{span}\left\{\Psi_{0}, \Psi_{0}, \Psi_{0}\right\}$. One of the subcases is $\Pi=\operatorname{span}\left\{\phi \otimes \psi_{1}, \phi \otimes\right.$ $\left.\psi_{2}, \phi \otimes \psi_{3}\right\}$. In this case, $w_{1}=u_{11} \phi \otimes \psi_{1}+u_{12} \phi \otimes \psi_{2}+u_{13} \phi \otimes \psi_{3}, w_{2}=u_{21} \phi \otimes \psi_{1}+$ $u_{22} \phi \otimes \psi_{2}+u_{23} \phi \otimes \psi_{3}$ and $w_{3}=u_{31} \phi \otimes \psi_{1}+u_{32} \phi \otimes \psi_{2}+u_{33} \phi \otimes \psi_{3}$, where the matrix $\left[u_{i j}\right]$ has rank three, since $w_{1}, w_{2}$ and $w_{3}$ are linearly independent. We choose the ILOs $F^{[1]}, F^{[2]}$ and $F^{[3]}$ such that

$$
\begin{gathered}
F_{1}^{[1]}\left(v_{1}\right)=\frac{1}{\sigma_{1}} e_{1}, \quad F_{1}^{[1]}\left(v_{2}\right)=\frac{1}{\sigma_{2}} e_{2}, \quad F_{1}^{[1]}\left(v_{3}\right)=\frac{1}{\sigma_{3}} e_{3}, \quad F_{2}^{[1]}=\left(\begin{array}{ccc}
u_{11}^{*} & u_{12}^{*} & u_{13}^{*} \\
u_{21}^{*} & u_{22}^{*} & u_{23}^{*} \\
u_{31}^{*} & u_{32}^{*} & u_{33}^{*}
\end{array}\right)^{-1}, \\
F^{[1]}=F_{2}^{[1]} F_{1}^{[1]}, \quad F^{[2]}(\phi)=e_{1}, \quad F^{[3]}\left(\psi_{1}\right)=e_{1}, \quad F^{[3]}\left(\psi_{2}\right)=e_{2}, \quad F^{[3]}\left(\psi_{2}\right)=e_{2} .
\end{gathered}
$$


Then the new coefficient matrix is

$$
\begin{aligned}
& \bar{C}=F_{2}^{[1]}\left(\begin{array}{ccc}
\frac{1}{\sigma_{1}} & 0 & 0 \\
0 & \frac{1}{\sigma_{2}} & 0 \\
0 & 0 & \frac{1}{\sigma_{3}}
\end{array}\right)\left(\begin{array}{ccc}
\sigma_{1} & 0 & 0 \\
0 & \sigma_{2} & 0 \\
0 & 0 & \sigma_{3}
\end{array}\right)\left(\begin{array}{ccccccccc}
u_{11}^{*} & u_{12}^{*} & u_{13}^{*} & 0 & 0 & 0 & 0 & 0 & 0 \\
u_{21}^{*} & u_{22}^{*} & u_{23}^{*} & 0 & 0 & 0 & 0 & 0 & 0 \\
u_{31}^{*} & u_{32}^{*} & u_{33}^{*} & 0 & 0 & 0 & 0 & 0 & 0
\end{array}\right) \\
& =\left(\begin{array}{lllllllll}
1 & 0 & 0 & 0 & 0 & 0 & 0 & 0 & 0 \\
0 & 1 & 0 & 0 & 0 & 0 & 0 & 0 & 0 \\
0 & 0 & 1 & 0 & 0 & 0 & 0 & 0 & 0
\end{array}\right)
\end{aligned}
$$

which corresponds to the state $|000\rangle+|101\rangle+|202\rangle$.

By investigating the rest cases similarly, all together we have the following canonical

\begin{tabular}{|c|c|}
\hline Class & Canonical Vector \\
\hline $\operatorname{span}\left\{\Psi_{0}, \Psi_{0}, \Psi_{0}\right\}$ & $\begin{array}{ll}|000\rangle+|101\rangle+|202\rangle, & |000\rangle+|110\rangle+|220\rangle \\
|000\rangle+|111\rangle+|202\rangle, & |000\rangle+|111\rangle+|220\rangle \\
|000\rangle+|111\rangle+|201\rangle, & |000\rangle+|111\rangle+|222\rangle\end{array}$ \\
\hline $\operatorname{span}\left\{\Psi_{0}, \Psi_{0}, \Psi_{1}\right\}$ & $|000\rangle+|011\rangle+|1 \phi \varphi\rangle+|2 \chi \psi\rangle$ \\
\hline $\operatorname{span}\left\{\Psi_{0}, \Psi_{0}, \Psi_{2}\right\}$ & $\begin{array}{l}|000\rangle+|011\rangle+|022\rangle+|101\rangle+|202\rangle \\
|000\rangle+|011\rangle+|022\rangle+|110\rangle+|220\rangle \\
|000\rangle+|011\rangle+|022\rangle+|101\rangle+|212\rangle\end{array}$ \\
\hline $\operatorname{span}\left\{\Psi_{1}, \Psi_{1}, \Psi_{0}\right\}$ & $\begin{array}{l}|000\rangle+|011\rangle+|101\rangle+|112\rangle+|2 \phi \varphi\rangle \\
|000\rangle+|011\rangle+|112\rangle+|120\rangle+|2 \phi \varphi\rangle \\
|000\rangle+|011\rangle+|120\rangle+|101\rangle+|2 \phi \varphi\rangle \\
\end{array}$ \\
\hline $\operatorname{span}\left\{\Psi_{1}, \Psi_{1}, \Psi_{1}\right\}$ & $\begin{array}{l}|000\rangle+|011\rangle+|101\rangle+|112\rangle+|202\rangle+|221\rangle \\
|000\rangle+|011\rangle+|101\rangle+|112\rangle+|210\rangle+|202\rangle \\
|000\rangle+|011\rangle+|101\rangle+|112\rangle+|221\rangle+|210\rangle \\
|000\rangle+|011\rangle+|112\rangle+|120\rangle+|202\rangle+|221\rangle \\
|000\rangle+|011\rangle+|112\rangle+|120\rangle+|221\rangle+|210\rangle \\
|000\rangle+|011\rangle+|120\rangle+|101\rangle+|221\rangle+|210\rangle \\
\end{array}$ \\
\hline $\operatorname{span}\left\{\Psi_{1}, \Psi_{1}, \Psi_{2}\right\}$ & $\begin{array}{l}|000\rangle+|011\rangle+|022\rangle+|101\rangle+|112\rangle+|202\rangle+|221\rangle \\
|000\rangle+|011\rangle+|022\rangle+|101\rangle+|112\rangle+|210\rangle+|202\rangle \\
|000\rangle+|011\rangle+|022\rangle+|101\rangle+|112\rangle+|221\rangle+|210\rangle\end{array}$ \\
\hline $\operatorname{span}\left\{\Psi_{2}, \Psi_{1}, \Psi_{0}\right\}$ & $\begin{array}{l}|000\rangle+|011\rangle+|022\rangle+|101\rangle+|112\rangle+|202\rangle \\
|000\rangle+|011\rangle+|022\rangle+|101\rangle+|112\rangle+|220\rangle \\
|000\rangle+|011\rangle+|022\rangle+|101\rangle+|112\rangle+|221\rangle\end{array}$ \\
\hline
\end{tabular}
states under SLOCC:

where $\phi, \varphi, \chi, \psi$ are pure states in $\mathbb{C}^{3}$.

According to structures of the space $\Pi$, we have got that three qutrits can be entangled 
in twelve inequivalent ways under SLOCC, where the states in the class of $\operatorname{dim} \Pi=1$, the first two states in $\operatorname{span}\left\{\Psi_{0}, \Psi_{0}\right\}$ and $\operatorname{span}\left\{\Psi_{0}, \Psi_{0}, \Psi_{0}\right\}$ are either fully or bi-separable.

\section{Conclusion and Remarks}

We have shown that entangled states of two and three qutrits can be classified into two and twelve equivalent types respectively under stochastic local operations and classical communication, based upon the analysis of the structure of the right singular subspace of the coefficient matrix of the states in an arbitrary conical product basis.

The range criterion [9], which can judge whether two pure states are inequivalent under SLOCC, classifies multipartite entanglement by analyzing the structure of the ranges of the states. In fact, a closer study we can get that the range of a state is equivalent to the right singular subspace of the state. As the ways of entanglement are concerned, the result of theorem 2 in [9] is included in section 3.2.

The way of classifying pure states under SLOCC can be generalized to high dimensional case by investigating the structures of the space $\Pi$. It is easily seen that there are $n$ types of pure states in the space $\mathbb{C}^{n} \otimes \mathbb{C}^{n}$ under SLOCC. For pure stats in $\mathbb{C}^{n} \otimes \mathbb{C}^{n} \otimes \mathbb{C}^{n}$, one can also deal with their classification according to the dimension of right singular subspace. If the dimension of the subspace is 2 , there will be $C_{n}^{2}+(n-1)$ families of entanglement; If the dimension of the subspace is 3 , then $C_{n}^{3}+2\left(C_{n}^{2}-1\right)+(n-1)$ families; ...; If the dimension of the subspace is $n-1$, then $C_{n}^{n-1}+(n-2)\left(C_{n}^{n-2}-1\right)+\ldots+2\left(C_{n}^{2}-1\right)+(n-1)$ families. All together the number of the classification is $(n-1)^{2}+\sum_{i=2}^{n}\left[(1+i(n-i)) C_{n}^{n-i}-i(n-i)\right]$,

where $C_{n}^{n-i}=n ! / i !(n-i)$ !. For instance for $n=2$, we have that, the result of [8], two qubits can be entangled in two inequivalent ways.

\section{References}

[1] D. Kaslikowski et al., Phys. Rev. Lett. 85, 4418 (2000).

[2] D. Collins et al., Phys. Rev. Lett. 88, 040404 (2002).

[3] F. Pan and G.Y. Lu, Int. J. Mod. Phys. B 20, 1333 (2006)

[4] H. Bechmann-Pasquinucci and A. Peres, Phys. Rev. Lett. 85, 3313 (2000).

[5] M. Bourennane, A. Karlsson, and G. Björk, Phys. Rev. A 64, 012306 (2001). 
[6] N. J. Cerf, M. Bourennane, A. Karlsson, and N. Gisin, Phys. Rev. Lett. 88, 127902 (2002).

[7] F. Verstraete, J. Dehaene, B. De Moor and H. Verschelde, Phys. Rev. A 65, 052112 (2002).

[8] W. Dür, G. Vidal and J. I. Cirac, Phys. Rev. A 62, 062314 (2000).

[9] L. Chen and Y.X. Chen, Phys. Rev. A 73, 052310(2006).

[10] A. Miyake and F. Verstraete, Phys. Rev. A 69, 012101(2004).

[11] D.F. Li, X.R. Li, H.T. Huang, X.X. Li, Phys. Lett. A 359, 428-237 (2006).

[12] F. Verstraete, J. Dehaene, B. De Moor and H. Verschelde, Phys. Rev. A 65, 052112 (2002).

[13] M.F. Cornelio and A. F. R. de Toledo Piza, Phys. Rev. A 73, 032314 (2006).

[14] Y.M. Di and Y. Cao, Commun. Theor. Phys. 45, 596 (2006).

[15] L. Lamata, J. León, D. Salgado and E. Solano, Phys. Rev. A 74, 052336 (2006); Phys. Rev. A 75, 022318 (2007).

[16] J.G. Luque and J.Y. Thibon, Phys. Rev. A 67, 042303 (2003); J. Phys. A 39, 371-377 (2006).

[17] P. Levay, Phys. Rev. A 71012334 (2005); J. Phys. A 399533 (2006).

[18] A. Miyake, Phys. Rev. A 67, 012108 (2003).

A. Miyake and M. Wadati, Quant. Inf. Comp. 2, 540 (2002). 